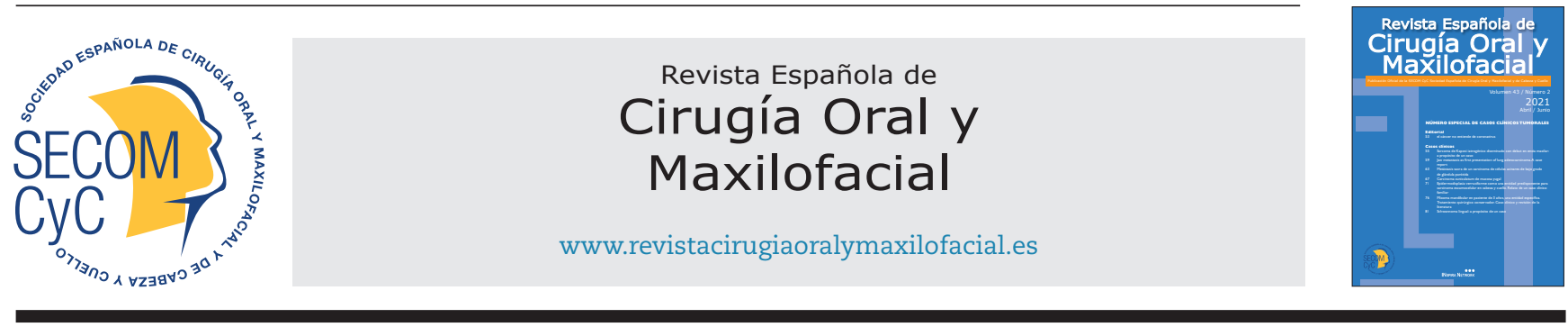

Caso clínico

\title{
Epidermodisplasia verruciforme como una entidad predisponente para carcinoma escamocelular en cabeza y cuello. Relato de un caso clínico familiar
}

\section{Galo Fernando Guzmán Castillo ${ }^{1}$, Judith Natalia Vásconez Escobar², Elio Fabio Sánchez Cortes ${ }^{3}$, Andrey Moreno Torres ${ }^{4}$ y Juan Pablo Gnecco Stouvenel ${ }^{1}$}

${ }^{1}$ Servicio de Cirugía Maxilofacial. Instituto Nacional de Cancerología. Pontificia Universidad Javeriana. Bogotá, Colombia. ${ }^{2}$ Especialista en Entrenamiento de Cirugía de Cabeza y Cuello. Instituto Nacional de Cancerología. Bogotá, Colombia. ${ }^{3}$ Especialista en Entrenamiento de Cirugía Oncológica. Instituto Nacional de Cancerología. Bogotá, Colombia. ${ }^{4}$ Servicio de Cabeza y Cuello. Instituto Nacional de Cancerología. Bogotá, Colombia

INFORMACIÓN DEL ARTÍCULO

Historia del artículo:

Recibido: 11 de febrero de 2021

Aceptado: 11 de julio de 2021

\section{Palabras clave:}

Epidermodisplasia verruciforme, carcinoma escamocelular, genodermatosis, virus del papiloma humano.

\section{R E S U M E N}

La epidermodisplasia verruciforme (EV) es una genodermatosis rara, caracterizada por producir susceptibilidad a la infección por el virus del papiloma humano (VPH) y la consecuente aparición de tumores malignos en la piel expuesta al sol. Dentro de estos, el más frecuente es el carcinoma escamocelular.

Presentamos un reporte de caso familiar con el antecedente de EV de dos hermanos que se encontraban en estadios avanzados de carcinoma escamocelular en región facial, además de lesiones en miembros superiores, quienes fueron sometidos a resección quirúrgica amplia, vaciamiento linfático ganglionar cervical y reconstrucción con colgajos libres mediante microcirugía.
Keywords:

Epidermodysplasia verruciformis, squamous cell carcinoma, human papilomavirus, genodermatosis.
Epidermodysplasia verruciformis as a predisposal entity for squamous cell carcinoma. A familial case report

\section{A B S T R A C T}

Epidermodysplasia verruciformis is a rare genodermatosis, in which there is an increased susceptibility for human papillomavirus (HPV) infection and the subsequent apparition of malignant tumours, being the squamous cell carcinoma the most frequent of those. We present this familiar case report of two male siblings who presented advanced squamous cell carcinoma in the facial area and lesions in arms and forearms, who were taken to major surgical resection, cervical lymph node resection and microvascular free flap reconstruction.

\footnotetext{
${ }^{*}$ Autor para correspondencia:

Correo electrónico: judithvasconez@yahoo.com (Judith Natalia Vásconez Escobar).

DOI: 10.20986/recom.2021.1261/2021
}

1130-0558/@ 2021 SECOM CyC. Publicado por Inspira Network. Este es un artículo Open Access bajo la licencia CC BY-NC-ND (http://creativecommons.org/licenses/by-nc-nd/4.0/). 


\section{INTRODUCCIÓN}

La epidermodisplasia verruciforme (EV) es una entidad rara, autosómica recesiva. Presenta una prevalencia menor de 1 caso por 1.000 .000 de habitantes. Fue descrito por F. Lewandowsky y W. Lutz en el año 1922, con el caso de una paciente de 29 años, quien presentaba lesiones cutáneas generalizadas de aspecto verrucoso e irregular, por lo cual la nombraron como epidermodisplasia verruciforme ${ }^{1}$.

La susceptibilidad a la infección por el virus del papiloma humano (VPH) se encuentra relacionada con la EV. Se han relacionado que los VPH-5 y VPH-8 son los subtipos que se asocian con la EV². Esta infección causa inmunodeficiencia cutánea en la $\mathrm{EV}$, lo que predispone a presentar lesiones benignas y malignas, especialmente carcinoma escamocelular (CE) de piel en regiones expuestas al sol3. El desarrollo de otros tipos de EV está relacionado con inmunodeficiencia por trasplante renal, enfermedades de Hodgkin, lupus eritematoso sistémico y VIH ${ }^{4}$.

Las alteraciones genéticas se relacionan con los genes EVER, especialmente EVER1/TMC6 y EVER2/TMC8. La proliferación de queratinocitos infectados está ligada con la pérdida de función de estos dos genes, también en la replicación viral en las células del huésped ${ }^{5}$. Otros genes, como $\mathrm{RHOH}$, LCK, MST1, CORO1A, IL7, RASGRP1, CARMIL2, TPP2, DCLRE1C y DOCK8, están ligados a esta entidad. La inmunodeficiencia primaria está vinculada a la alteración sistémica de células T y la incapacidad de protección a la infección viral 5 .

Las lesiones cutáneas inducidas por la EV relacionada con VPH es polimórfica6 ${ }^{6}$ La manifestación clínica inicia generalmente en la infancia y consiste en placas delgadas similares a la pitiriasis versicolor, además de verrugas planas ${ }^{5}$. Las lesiones en extremidades y cara son planas, de aspecto verrugoso. Las lesiones torácicas tienen aspecto de placas con bordes rojo parduzco ${ }^{6}$.

Histológicamente presentan un patrón citopático, que consiste en la clarificación del citoplasma, pequeños núcleos picnóticos y gránulos basófilos en las capas superiores ${ }^{7}$. El carcinoma escamocelular en la EV se caracteriza por invasión de la dermis por células epidérmicas, en algunos casos con características de la enfermedad de Bowen.

La EV se ha clasificado basándose en la patogénesis y su presentación clínica en dos categorías: genética y adquirida. La EV genética, a su vez, se divide en dos categorías: la primera denominada "clásica" está asociada con la mutación con los genes EVER1/TMC6 y EVER2/TMC8 y la "No clásica" relacionada con otros genes como RHOH, MST1, CORO1A y ECM1. El gen TMC8 codifica una proteína similar a un canal transmembrana; las mutaciones en este gen se relacionan con epidermodisplasia verruciforme tipo 2 (EV2), que es de característica autosómico recesiva ${ }^{8}$.

La EV adquirida se define en pacientes con compromiso secundario en el sistema inmunológico y subdividida en EV asociada con inmunodeficiencia por VIH y asociada con causas iatrogénicas, como medicamentos inmunosupresores ${ }^{9}$.

\section{CASOS CLÍNICOS}

Se reporta un caso familiar de dos hermanos que presentaban el antecedente paterno de EV. Los mismos fueron trata- dos durante años de manera conservadora, obteniendo malos resultados y progresión locorregional de su enfermedad; requirieron resecciones amplias con reconstrucción con colgajo libre microvascular.

- Caso clínico 1: paciente masculino de 48 años, cuadro clínico de 8 años de evolución, con manifestación clínica de verrugas planas en región de canto externo izquierdo, con resección previa de la lesión hace 4 años; recibió 35 sesiones de radioterapia.

Presentaba múltiples placas eritematosas irregulares asociado a verrugas planas tipo pitiriasiformes en miembros superiores y abdomen. En la región frontal presentó múltiples placas eritematosas de aspecto cicatricial, con superficie queratósica, la de mayor tamaño de $20 \mathrm{x}$ $20 \mathrm{~mm}$. En canto externo ojo izquierdo presentaba un tumor adherido a planos profundos de 40 x $23 \mathrm{~mm}$, doloroso a la palpación. En dorso de mano izquierda presentaba placa indurada de 18 x $20 \mathrm{~mm}$. Globo ocular izquierdo con disminución de agudeza visual y diplopía a supraducción.

La biopsia con resultado de carcinoma escamocelular infiltrante de célula grande queratinizante moderadamente diferenciado, con nivel de invasión a dermis reticular (IV), sin invasión linfovascular identificada (Figura 1). La tomografía computarizada (TC) evidencia compromiso de órbita izquierda con infiltración a músculos extraoculares, ojo izquierdo (Figura 1).

Se realizó maxilectomía de supraestructura, con exenteración orbitaria izquierda, parotidectomía superficial izquierda, vaciamiento radical modificado izquierdo y reconstrucción con colgajo libre anterolateral de muslo con anastomosis en arteria y vena facial izquierda (Figura 2).

- Caso clínico 2: paciente masculino de 52 años, con antecedente de carcinomas no melanomas en piel, tratamiento previo con capecitabina y 5-fluorouracilo, múltiples resecciones de carcinomas en piel. Presentó una lesión ulcerada sobreelevada, con fácil sangrado en la región frontal izquierda. El resultado de la biopsia indicó carcinoma escamocelular infiltrante de célula grande queratinizante moderadamente diferenciado (Figura 3). La TC evidencia compromiso de región frontal sin infiltración de cortical externa de hueso frontal, por lo que se realizó resección de subunidad frontal sin compromiso óseo (Figura 3).

En los dos casos se realizan controles a los 6 meses, sin evidencia de recaída tumoral. Se registran fotografías clínicas postoperatorias (Figura 4).

El cariotipo de ambos pacientes reportó en los análisis, mediante banda $\mathrm{Q}$ y banda $\mathrm{G}$, una constitución cromosómica normal y en el análisis genético en asociación con epidermoplasia (TMC6, NDP, TMC8, IL7, CIB1, ERCC4, ERCC5, XPA, XPC, DDB2) se encontró una variante patogénica en homocigosis del gen TMC8 en variante NM_152468.5(TMC8):c.1084G>T p.(Glu362*) en ambos hermanos. De acuerdo con las recomendaciones del American College of Genetics and Genomics (AMGG), esta variante es clasificada como patogénica8.

La variante NM_152468.5(TMC8):c.1084G>T p.(Glu362*) ha sido descrita en la literatura como la responsable de provocar un codón de parada prematuro, que se espera genere una pro- 

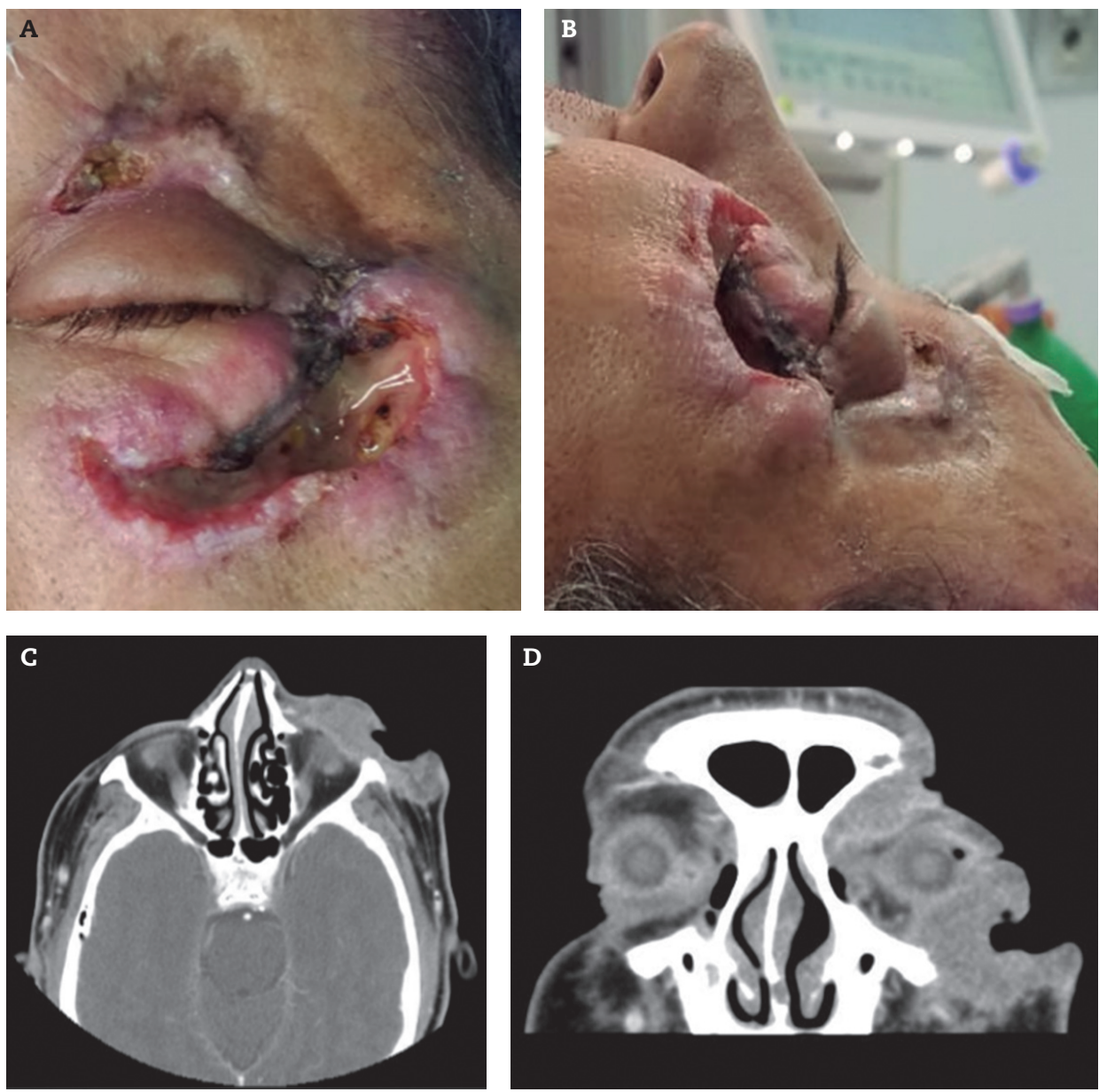

Figura 1. Presentación clínica de tumoración en región de canto externo izquierdo. A: vista frontal. B: vista. lateral. C: TC corte axial. D: corte coronal.
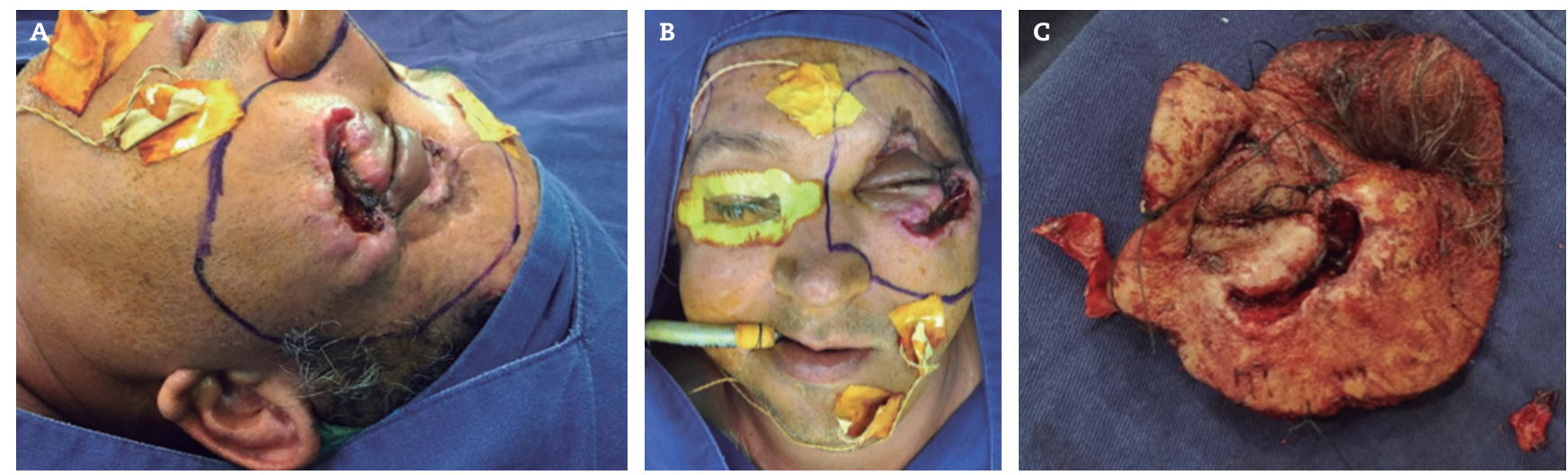

Figura 2. Márgenes de resección y espécimen quirúrgico.

teína truncada o ausente, y se ha tomado la EV como modelo para identificar aquellos locus que aumenten la susceptibilidad

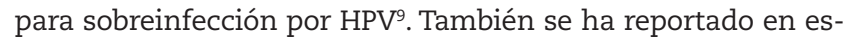
tado de homocigosis en individuos de una misma familia afectados por EV, como es el caso de nuestros pacientes. Además se ha encontrado en la base de datos ClinVar que esta mutación puede clasificarse como factor de riesgo (ID: 4747) para EV ${ }^{10}$.

\section{DISCUSIÓN}

La epidermodisplasia verruciforme (EV) es una genodermatosis autosómica recesiva poco frecuente ${ }^{1}$, que involucra al sistema inmunológico. Se caracteriza por un amplio espectro clínico relacionado con la infección por el VPH y la pre- 

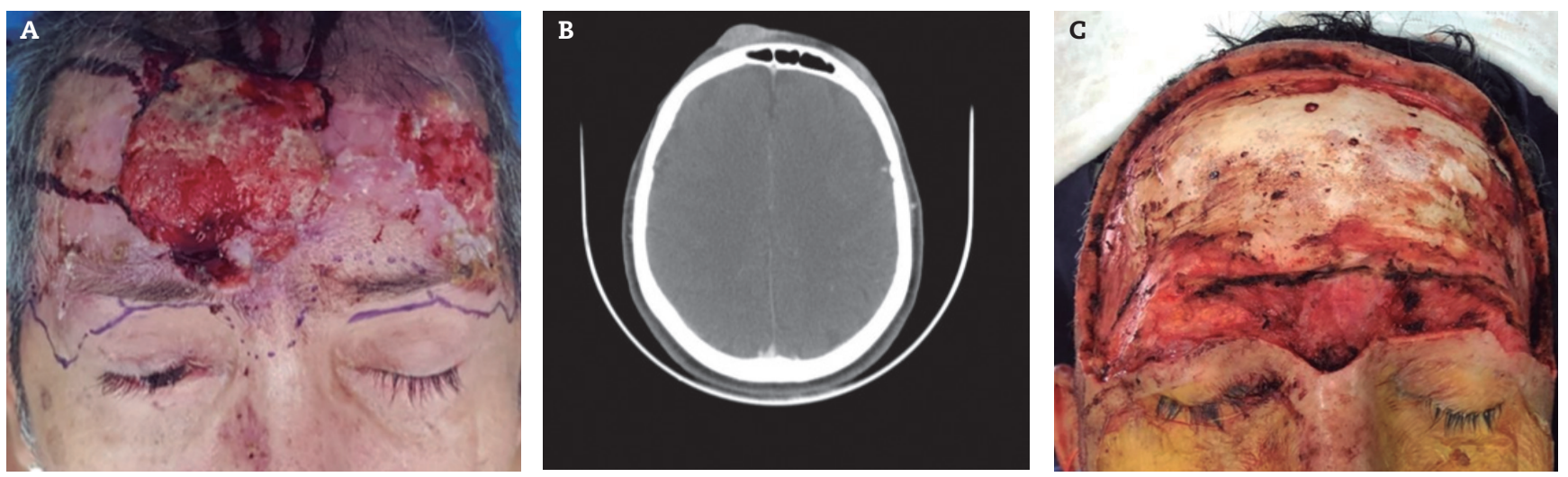

Figura 3. A: lesión preoperatoria región frontal. B: TC evidencia lesión región frontal. C: defecto intraoperatorio.
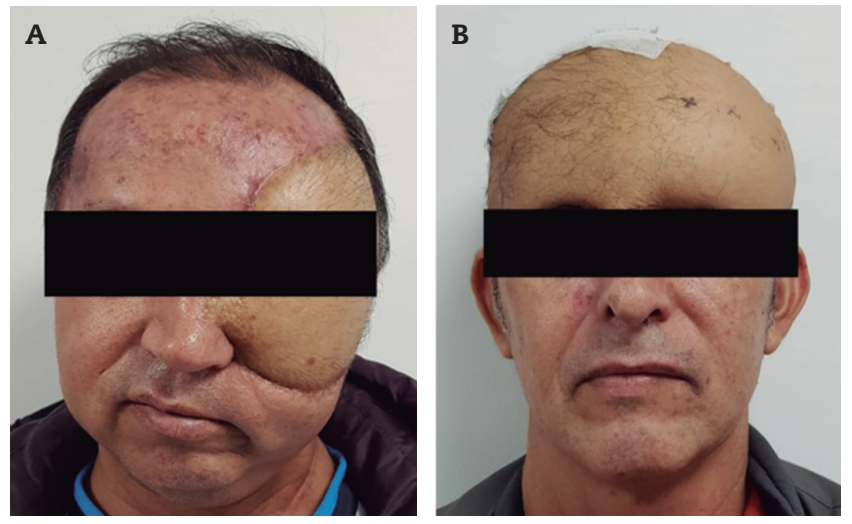

dioterapia adyuvante, dada la posibilidad de progresión de nuevas lesiones de carcinoma escamocelular ${ }^{14}$. El seguimiento por dermatología, el uso de foto-protección y la consejería genética al núcleo familiar son los pilares fundamentales para detectar aquellas lesiones de alto riesgo de forma temprana, y tratarlas adecuadamente en todos los miembros de la familia.

\section{CONFLICTO DE INTERESES}

Los autores declaran no tener conflicto de intereses.

Figura 4. Resultados postoperatorios. A: paciente 1. B: paciente 2 .

B I B L I O G R A F Í A

1. Lewandowsky F, Lutz W. Ein Fall einer bisher nicht beschriebenen Hauterkrankungn (Epidermodysplasia verruciformis). Arch Dermatol Syphilol 1922;12:306-16

disposición a presentar lesiones, especialmente carcinoma escamocelular de piel en regiones expuestas al sol ${ }^{5}$. En los pacientes presentados en este artículo se encontró una variante patogénica del gen TMC8 en los dos hermanos, con características clínicas y patológicas similares desde edad temprana, que fueron manejados como lesiones benignas y con poco seguimiento, lo que empeoró la evolución de los casos. Esto está en relación con las mutaciones descritas por Huang y cols., siendo el caso de nuestros pacientes EV de tipo genético con variante clásica, que tiene como característica la aparición de lesiones tempranas con alto riesgo de desarrollar cáncer de piel no melanoma, tal como carcinoma escamocelular en un $30-70 \%{ }^{11}$.

Actualmente no hay tratamiento curativo, Se ha descrito $^{12}$ que las lesiones tempranas pueden tratarse con crioterapia, las verrugas planas con retinoides, imiquimod o 5-FU con algunos resultados prometedores ${ }^{13}$; sin embargo, al tener lesiones que progresan (transformación a carcinoma escamocelular), a pesar de tratamiento se recomienda resección quirúrgica con márgenes amplios y reconstrucciones según el defecto con colgajos locales, regionales o microvasculares, como fue el caso de nuestros pacientes.

Estos pacientes requieren seguimiento a largo plazo ante el alto riesgo de aparición de nuevas lesiones, ya que, por la condición de piel, no se recomienda administración de ra-

2. Przybyszewska J, Zlotogorski A, Ramot Y. Re-evaluation of epidermodysplasia verruciformis: Reconciling more than 90 years of debate. J Am Acad Dermatol. 2017 Jun;76(6):11611175. doi: 10.1016/j.jaad.2016.12.035

3. Gül U, Kiliç A, Gönül M, Cakmak SK, Bayis SS. Clinical aspects of epidermodysplasia verruciformis and review of the literature. Int J Dermatol. 2007;46(10):1069-72. DOI: 10.1111/ j.13654632.2006.03014.x

4. Youssefian L, Vahidnezhad H, Mahmoudi H, Saeidian AH, Daneshpazhooh M, Kamyab Hesari K, et al. Epidermodysplasia Verruciformis: Genetic Heterogeneity and EVER1 and EVER2 Mutations Revealed by Genome-Wide Analysis. J Invest Dermatol. 2019;139(1):241-4. DOI: 10.1016/j.jid.2018.07.010.

5. Majewski S, Jabło囚ska S. Epidermodysplasia verruciformis as a model of human papillomavirus-induced genetic cancer of the skin. Arch Dermatol. 1995;131(11):1312-8.

6. De Oliveira WR, Festa Neto C, Rady PL, Tyring SK. Clinical aspects of epidermodysplasia verruciformis. J Eur Acad Dermatol Venereol. 2003;17(4):394-8. DOI: 10.1046/j.1468-3083. 2003.00703.x.

7. Ramoz N, Rueda LA, Bouadjar B, Montoya LS, Orth G, Favre M. Mutations in two adjacent novel genes are associated with epidermodysplasia verruciformis. Nat Genet. 2002;32(4):57981. DOI: $10.1038 / \mathrm{ng} 1044$.

8. Richards S, Aziz N, Bale S, Bick D, Das S, Gastier-Foster J, et al. ACMG Laboratory Quality Assurance Committee. Standards and guidelines for the interpretation of sequence variants: a joint consensus recommendation of the American College of Medical 
Genetics and Genomics and the Association for Molecular Pathology. Genet Med. 2015;17(5):405-24. DOI: 10.1038/gim.2015.30.

9. Ramoz N, Rueda LA, Bouadjar B, Favre M, Orth G. A susceptibility locus for epidermodysplasia verruciformis, an abnormal predisposition to infection with the oncogenic human papillomavirus type 5, maps to chromosome 17qter in a region containing a psoriasis locus. J Invest Dermatol. 1999;112(3):25963. DOI: 10.1046/j.1523-1747.1999.00536.x.

10. National Center for Biotechnology Information. ClinVar; [VCV000004747.2]. Disponible en: https://www.ncbi.nlm.nih. gov/clinvar/variation/4747/

11. Huang S, Wu JH, Lewis DJ, Rady PL, Tyring SK. A novel approach to the classification of epidermodysplasia verruciformis. Int J Dermatol. 2018;57(11):1344-50. DOI: 10.1111/ijd.14196.
12. Schierbeck J, Vestergaard T, Bygum A. Skin Cancer Associated Genodermatoses: A Literature Review. Acta Derm Venereol. 2019;99(4):360-9. DOI: 10.2340/00015555-3123.

13. Gupta AK, Davey V, Mcphail H. Evaluation of the effectiveness of imiquimod and 5-fluorouracil for the treatment of actinic keratosis: Critical review and meta-analysis of efficacy studies. J Cutan Med Surg. 2005;9(5):209-14. DOI: 10.1007/s10227-0050148-6.

14. De Oliveira WR, da Cruz Silva LL, Neto CF, Tyring S. Deleterious Effect of Radiation Therapy on Epidermodysplasia Verruciformis Patients. J Cutan Med Surg. 2015;19(4):416-21. DOI: 10.1177/1203475415576859.Figura 2. Márgenes de resección y espécimen quirúrgico. 